\title{
Automata-based Pattern Mining from Imperfect Traces
}

\author{
Giles Reger \\ University of Manchester \\ Oxford Road, M13 9PL \\ Manchester, UK \\ regerg@cs.man.ac.uk*
}

\author{
Howard Barringer \\ University of Manchester \\ Oxford Road, M13 9PL \\ Manchester, UK \\ howard@cs.man.ac.uk
}

\author{
David Rydeheard \\ University of Manchester \\ Oxford Road, M13 9PL \\ Manchester, UK \\ david@cs.man.ac.uk
}

\begin{abstract}
This paper considers automata-based pattern mining techniques for extracting specifications from runtime traces and suggests a novel extension that allows these techniques to work with so-called imperfect traces i.e. traces that do not exactly satisfy the intended specification of the system that produced them. We show that by taking a so-called edit-distance between an input trace and the language of a pattern we can extract specifications from imperfect traces and identify the parts of an input trace that do not satisfy the mined specification, thus aiding the identification and location of errors in programs.
\end{abstract}

\section{Keywords}

Pattern Mining, Specification Mining

\section{INTRODUCTION}

Formal program specifications are useful for a number of activities but they are often missing or incomplete. The field of specification mining $[13,18]$ aims to automatically construct formal program specifications from program artifacts. In this work we consider techniques that operate on program traces i.e. finite sequences of events that occur whilst a program is running.

One such approach $[19,5,6]$ uses a set of template patterns to detect predefined behaviours and then combine these together to form a specification. Thse regular patterns are described via automata, which allows for efficient checking. For example, consider the following pattern over the metasymbols $a$ and $b$ - shaded states are accepting states.

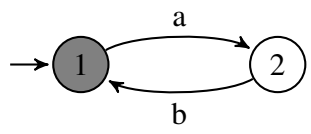

We can apply this pattern to the following trace by considering six instantiations, with each pair of symbols in the trace instantiating the pattern.

connect.open.close

We detect three patterns (1) $[a \mapsto$ connect, $b \mapsto$ open $]$, (2) $[a \mapsto$ connect, $b \mapsto$ close $]$ and (3) $[a \mapsto$ open, $b \mapsto$ close $]$. These can then be "combined" to form a larger specification:

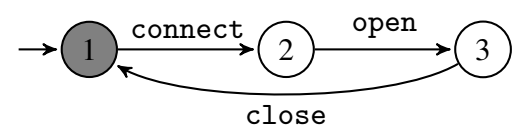

This general approach can be used with different patterns and methods of pattern combination. However, it has a major drawback - imagine if

${ }^{*}$ Part of this work was supported by the Engineering and Physical Sciences Research Council [grant number EP/P505208/1]. we had a trace with the above sequence repeated a thousand times followed by the two events connect and open i.e. missing the final close. We would fail to detect the two patterns involving close and therefore not extract the above specification. The problem is that this approach assumes perfect traces i.e. that the correct behaviour is contained within the given traces. This assumption is unrealistic - we would like to be able to deal with cases where there are small errors in traces. The notion is that a programming pattern may hold for the majority of a program but the program may contain one or two bugs.

One approach [5] to dealing with this issue is to reset a pattern being checked to its initial state when an error occurs - but this technique would not detect the required patterns in our above example. Instead we want to be able to measure how closely a trace matches a pattern. This paper presents an approach that extends the automta-based pattern mining approach to imperfect traces by considering so-called edit distances between a trace and a pattern's language.

This work is motivated by the notion that automata-based pattern-mining is a desirable approach to specification mining but to make it applicable we need to deal with the issue of imperfect traces. The main advantage of the pattern-based approach is that we can use arbitrarily complex patterns, unlike techniques based on data-mining, allowing us us to develop more sound combination strategies (described in [17]) and consider further work (discussed in Section 9) that involves extensions to the patternmining approach that could not be applied to alternative approaches.

Structure. Section 2 formally introduces the concept of pattern checking and composition. Section 3 discusses methods for dealing with the imperfect traces problem and Sections 4, 5 and 6 present our proposed solutions. Section 7 presents two experiments and Section 8 discusses related work. Finally, we conclude in Section 9.

\section{PATTERN CHECKING}

In this section, we introduce a pattern checking framework by first describing how patterns are extracted from traces, then considering how this can be done efficiently and finally discussing how extracted patterns are combined.

\subsection{Checking patterns}

In this account, a pattern is a regular language over symbols i.e. a set of traces (finite sequences) of symbols. We consider patterns as automata:

Definition 1 (PATTERN). A pattern $p=\left\langle Q, \Sigma, \delta, q_{0}, F\right\rangle$ is an automaton where $Q$ is a finite set of states, $\Sigma$ is a finite alphabet of symbols, $\delta \in Q \times \Sigma \rightarrow Q$ is a transition function, $q_{0} \in Q$ is an initial state and $F \subseteq Q$ is a set of accepting states. The language of a pattern, $\mathcal{L}(p)$ is the set of traces it accepts i.e. $\tau \in \mathcal{L}(p)$ iff there exists a path $q_{0} \stackrel{\tau}{\rightarrow} q$ and $q \in F$ where $\rightarrow$ is $\delta$ lifted to traces. 
The process of checking a pattern against a trace considers all possible combinations of symbols in the trace as replacements for the pattern's current symbols. To replace a pattern's symbols we instantiate it.

DEFINITION 2 (INSTANTIATION). Given a pattern $p$ and a map $\varphi$ from $p . \Sigma$ to $\Sigma^{\prime}$, the instantiated pattern $\varphi(p)$ has alphabet $\Sigma^{\prime}$ and is the result of applying $\varphi$ to every symbol in $p$.

The checking process then checks if each particular instantiation of the pattern holds on the trace. We say an instantiated pattern holds on a trace if the trace appears in the instantiated pattern's language after we remove irrelevant symbols. To remove irrelevant symbols we project the trace.

DEFINITION 3 (PROJECTION). The projection $\pi_{\Sigma}(\tau)$ of trace $\tau$ over alphabet $\Sigma$ is defined as $\tau$ with all elements not in $\Sigma$ removed.

Therefore, the detected instantiated patterns are given as follows.

DEFINITION 4 (EXTRACTED PATTERNS). Given a pattern $p$ and trace $\tau$ the extracted patterns $\operatorname{detect}(p, \tau)$ are

$$
\left\{\varphi(p) \mid \forall a \in p . \Sigma: \varphi(a) \in \tau \wedge \pi_{\varphi(p) . \Sigma}(\tau) \in \mathcal{L}(\varphi(p))\right\}
$$

\subsection{Checking patterns efficiently}

We discuss two approaches that allow us to check patterns efficiently.

\subsubsection{Checking many instantiations}

For each pattern we need to check all possible instantiations. Typically we restrict this technique to patterns over 2 or 3 symbols (this does not restrict the number of symbols in the mined specification). We can then compute the extracted instantiated patterns for a pattern using a 2 or 3 dimensional grid of reached states - this approach was first used in [19]. For the introductory example the following matrix would represent the states reached in the pattern after checking the trace (- means failure).

\begin{tabular}{|c|c|c|c|c|}
\hline & \multicolumn{3}{|c|}{ a } \\
\hline & & connect & open & close \\
\hline \multirow{3}{*}{ b } & connect & 2 & - & - \\
\hline & open & 1 & 2 & - \\
\hline & close & 1 & 1 & 2 \\
\hline
\end{tabular}

The restriction of patterns to 2 or 3 symbols is for efficiency reasons as this approach has space complexity $O\left(n^{m}\right)$ and time complexity $O\left(n^{m-1}|\tau|\right)$ given an alphabet of size $n$ and pattern with $m$ symbols. A more efficient symbolic approach using binary decision diagrams is explored in [6]

\subsubsection{Checking many patterns}

If we want to check multiple patterns we would currently need to repeat the above process multiple times i.e. for each pattern. However, given a set of patterns with the same set of symbols we can construct a pattern checker that checks all these patterns simultaneously by taking the union of the patterns and labeling states with the patterns that are accepting at that state. This approach was previously presented in [17].

DEFINITION 5 (PATTERN CHECKER). Given an alphabet of symbols $\Sigma$ and a set of patterns $p_{1}, \ldots, p_{n}$ over $\Sigma$ let the pattern checker for these patterns be $\mathrm{C}\left(p_{1}, \ldots, p_{n}\right)=\langle Q, \Sigma, \Rightarrow, \Gamma\rangle$ where

$$
\begin{array}{ll}
Q & =p_{1} \cdot Q \times \ldots \times p_{n} \cdot Q \\
\Rightarrow\left(a,\left(q_{1}, \ldots, q_{n}\right)\right) & =\left(p_{1} . \delta\left(a, q_{1}\right), \ldots, p_{n} . \delta\left(a, q_{n}\right)\right) \\
\Gamma\left(\left(q_{1}, \ldots, q_{n}\right)\right) & =\left\{p_{i} \mid q_{i} \in p_{i} . F\right\}
\end{array}
$$

The patterns detected by pattern checker $\mathrm{C}$ in trace $\tau$ are therefore

$$
\mathrm{C}(\tau)=\left\{p \mid q_{0} \stackrel{\tau}{\Rightarrow} q \wedge p \in \Gamma(q)\right\}
$$

We can extend the notion of instantiation to pattern checkers and define extracted patterns for a pattern checker as follows.

DEFINITION 6 (PATTERn CHECKER EXTRACTED PATTERnS). Given a pattern checker $\mathrm{C}$ and trace $\tau$ the extracted patterns $\operatorname{detect}(\mathrm{C}, \tau)$ are

$$
\left\{p \mid \exists \varphi: \forall a \in p . \Sigma: \varphi(a) \in \tau \wedge p \in \varphi(\mathrm{C})\left(\pi_{\varphi(p) . \Sigma}(\tau)\right)\right\}
$$

For example, if we call the pattern in the introductory example $p_{1}$ and call the following pattern $p_{2}$

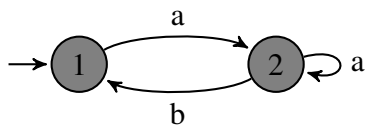

then the pattern checker for $p_{1}$ and $p_{2}$ would be

$$
\rightarrow\left\{p_{1}, p_{2}\right\} \stackrel{\mathrm{a}}{\longleftarrow \mathrm{b}}\left\{p_{2}\right\} \stackrel{\mathrm{a}}{\longrightarrow}\left\{p_{2}\right\} \stackrel{\mathrm{a}}{\stackrel{\mathrm{a}}{\rightleftarrows}}\left\{p_{2}\right\}
$$

where states are labeled using the output function $\Gamma$.

\subsection{Combining patterns}

The following is based on the technique introduced by Gabel and $\mathrm{Su}$ in [5]. Once we have extracted a set of patterns we can combine them together using standard automata intersection. However, this operation is only defined when two automata have the same alphabet. To give two automata the same alphabet we can expand them by placing self-looping transitions on each state for the missing symbols. For example, the three detected patterns from the introductory example become:

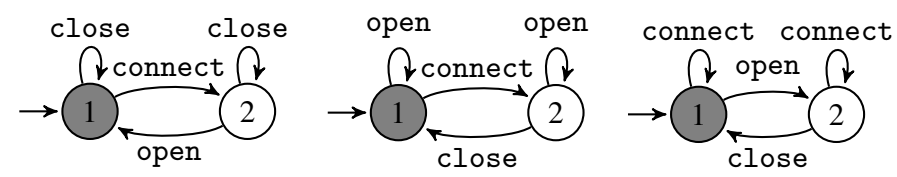

The intersection of these three patterns is the specification given in the introduction. Formally, combination is defined as follows.

DEFINITION 7 (COMBINATION). Given a set of instantiated patterns $p_{1}, \ldots, p_{n}$ with combined alphabet $\Sigma$, define their combination as combine $\left(p_{1}, \ldots, p_{n}\right)=\operatorname{expand}^{\Sigma \backslash p_{1} \cdot \Sigma}\left(p_{1}\right) \cap \ldots \cap \operatorname{expand}^{\Sigma \backslash p_{n} \cdot \Sigma}\left(p_{n}\right)$ where $\cap$ is automata intersection and expand ${ }^{\Sigma^{\prime}}$ is a function that adds self-looping transitions to a pattern for symbols in $\Sigma^{\prime}$.

We can either apply this combination operator or directly or use it to define specific combination rules. To use combination directly we can saturate the set by repeated application or extract a specification for each alphabet of events in the trace by combining together patterns with the same alphabet. However, this might be costly and not all extracted patterns necessarily contain useful information. Therefore, an alternative approach (which we do not consider further here) is to develop specific 
combination rules for given patterns, as is done in [5]. For example, they introduce the following sequencing rule for the pattern in our introductory example, which we will represent by the regular expression $(a b)^{*}$.

$$
\frac{\left(a \mathcal{L}_{1} b\right) *\left(b \mathcal{L}_{2} c\right) *(a c)^{*}}{\left(a \mathcal{L}_{1} b \mathcal{L}_{2} c\right)^{*}}
$$

We applied this by taking $\mathcal{L}_{1}=\mathcal{L}_{2}=\emptyset$. The use of $\mathcal{L}_{1}$ and $\mathcal{L}_{2}$ allows for repeated application of the rule. The closure of the set of extracted patterns with respect to a set of combination rules can then be computed.

\section{DEALING WITH IMPERFECT TRACES}

The previous framework will only detect a pattern if it matches exactly with an input trace. In this section we consider how it can be extended so that patterns are extracted if they match almost all of the input trace.

\subsection{What are imperfect traces?}

To say that a trace is "imperfect" we assume that there is an implicit specification that the program that produced the trace follows and there is some bug in the program that deviates from this specification. The process of specification mining is therefore to extract this implicit specification. Alternatively, the program might be correct but the trace recording process may be faulty - either way, identifying a specification and the trace imperfections can aid debugging efforts.

We could view these imperfections as uniform noise, however, in the case of programming bugs, it is likely that these imperfections are introduced by common mistakes such as forgetting to close a resource or check a condition, or accidentally calling the wrong method. We can therefore think of imperfections as small edits that involve the removal, addition or substitution of events from a 'perfect' trace.

\subsection{The restart approach}

Previous approaches (i.e.[6]) deal with imperfect traces by 'restarting' the pattern and counting the number of such restarts. With small patterns such as the alternation pattern ( $p_{1}$ from before) this can be effective. Let us consider the following common 3-symbol resource usage pattern.

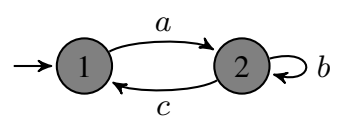

Consider checking the following (imperfect) trace for the instantiation $[a \mapsto$ open, $b \mapsto$ use, $c \mapsto$ close]. The checking would fail after the fifth event as an open event is omitted. If we restart here then we immediately fail again.

open.use.use.close.use.close.open.use.close

Instead, we would like to detect that the open event is missing and flag this as a potential bug.

Note that our proposed approach is a generalisation of the restart approach by making a substitution edit for the 'missing' symbol to reach the initial state.

\subsection{Edit distance}

As an alternative to the restart approach we consider replacing our previous condition that a trace must exactly match a pattern with the requirement that the edit-distance between the trace and any trace in the language of the pattern must be below some limit.

The edit-distance we consider uses the following "edit" operations: inserting a new symbol; deleting an existing symbol; substituting an existing symbol for a new symbol. The edit-distance between two traces is then given by the (minimum) number of edits that transform one trace into the other. This is sometimes called the Levenshtein distance [10]. Formally, this distance is given as follows.

DEFINITION 8 (LEVENSHTEIN DISTANCE). The Levenshtein distance between traces $\tau_{1}$ and $\tau_{2}$ is distance $\left(\tau_{1}, \tau_{2}\right)$, defined as

$$
\begin{aligned}
& \begin{array}{ll}
\text { distance }\left(\tau_{1}, \epsilon\right) & =\left|\tau_{1}\right| \\
\text { distance }\left(\epsilon, \tau_{2}\right) & =\left|\tau_{2}\right|
\end{array} \\
& \text { distance }\left(a \tau_{1}, b \tau_{2}\right)=\min \begin{cases}\operatorname{distance}\left(\tau_{1}, b \tau_{2}\right)+1 \\
\operatorname{distance}\left(a \tau_{1}, \tau_{2}\right)+1 \\
\operatorname{distance}\left(\tau_{1}, \tau_{2}\right)+1 & \text { if } a \neq b \\
\operatorname{distance}\left(\tau_{1}, \tau_{2}\right) & \text { if } a=b\end{cases}
\end{aligned}
$$

We define an updated notion of extracted patterns using this metric.

DEFINITION 9 (IMPERFECT EXTRACTED PATTERNS). Given a pattern $p$, trace $\tau$ and integer $\gamma>0$, which we call the tolerance, the imperfect extracted patterns imperfect_detect $(p, \tau, \gamma)$ are

$$
\left\{\begin{aligned}
\varphi(p) \mid & \forall a \in p . \Sigma: \varphi(a) \in \tau \wedge \\
& \exists \tau^{\prime} \in \mathcal{L}(\varphi(p)): \text { distance }\left(\tau^{\prime}, \pi_{\varphi(p) . \Sigma}(\tau)\right)<\gamma
\end{aligned}\right\}
$$

We extend this definition for pattern checkers as we did before (Sec. 2.2.2).

\subsection{Detecting bugs}

So far our approach has been abstract, considering traces of symbols generated by a program. But our motivation has been to extract specifications that allow us to detect potential bugs. To do so we need to be able to access information about the part of a program that generates a trace - we assume this is contained in a program trace.

DEFINITION 10 (PROGRAM TRACE). A program trace is a finite sequence of pairs of the form (code_point, event) where code_point identifies the point in the program that generates the event.

It is easy to extend our previous constructions to work on these program traces by ignoring the code point information. Our goal is to identify points in the program trace that should be 'edited' for a mined specification to hold. These edits will follow those described above i.e. the removal of an event, addition of an event between two existing events or replacement of one event with another. The solutions we describe in the following two sections will produce rewrites.

DEFINITION 11 (REWRITE). A rewrite $\rho$ is a finite sequence of indexes and rewrite operations that can be applied to a program trace to produce an 'edited' version. At index $i$ we write a deletion as $(i,-)$, insertion of event a as $(i,+a)$ and substitution with events a as $(i, \% a)$.

A rewrite can then be used to identify the code points that may contain bugs, and suggest potential solutions i.e. edits.

\section{EDITING ON FAILURE}

We first consider an approach that does not use the true edit-distance, but introduces a new 'restart' operation inspired by the metric. The idea is to introduce edit operations only when a trace fails to match a pattern.

\subsection{Failing-edit-distance}

In the following we introduce an alternative formulation of the edit-distance that only applies edits when we fail. We say a pattern fails for a trace if no extensions of the trace can satisfy the pattern. 


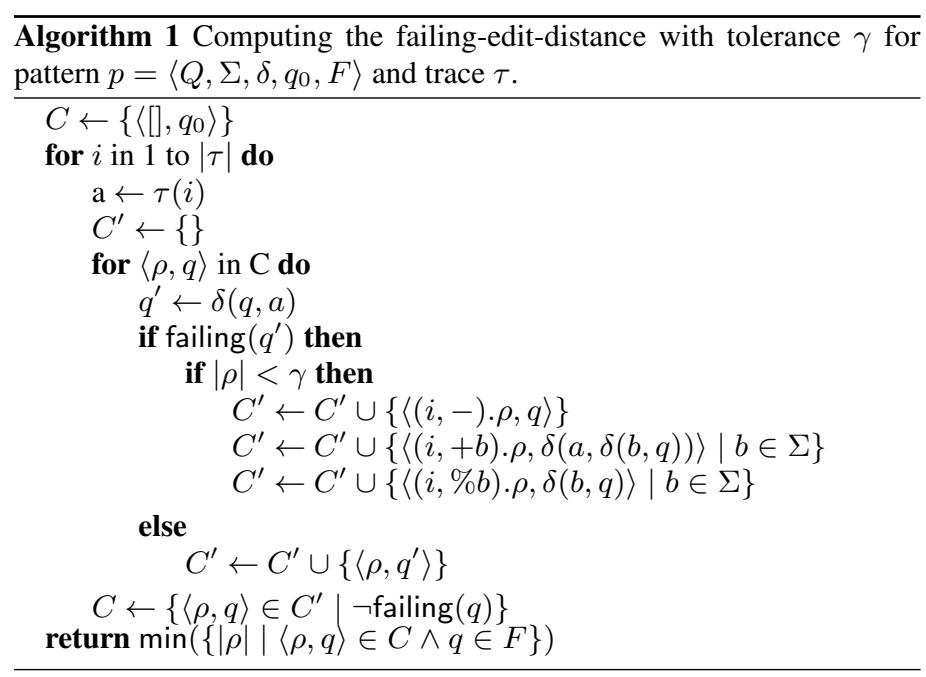

For pattern $p$ and trace $\tau$ let $\tau=\operatorname{good}(\tau) \cdot a \cdot \operatorname{rest}(\tau)$ where $\operatorname{good}(\tau)$ is longest prefix of $\tau$ such that there exists a trace $\tau^{\prime}$ such that $\operatorname{good}(\tau) \cdot \tau^{\prime} \in$ $\mathcal{L}(p)$ but for all traces $\tau^{\prime}$ we have $\operatorname{good}(\tau) . a . \tau^{\prime} \notin \mathcal{L}(p)$

Let edit be a function on symbols that non-deterministically replaces the symbol by the empty trace, a trace consisting of another symbol from the trace followed by the original symbol or another symbol in the trace i.e. it can pick one of the three edit operations discussed above.

An edited trace is defined recursively as

$$
\operatorname{edited}(\tau)=\begin{array}{ll}
\operatorname{edited}(\operatorname{good}(\tau) \cdot \operatorname{edit}(a) \cdot \operatorname{rest}(\tau)) & \text { if } \tau \notin \mathcal{L}(\tau) \\
\tau & \text { otherwise }
\end{array}
$$

i.e. the repeated application of the edit function to the event causing failure. As edit is non-deterministic the failing-edit-distance is given as the minimum number of times the edited function must be applied to a trace. This is still an edit-distance, but not necessarily minimal.

\subsection{Computing the failing-edit-distance}

To compute the failing-edit-distance we explore the non-deterministic edit operations by maintaining a number of possible configurations of the instantiated pattern. A configuration is a pair consisting of a rewrite (Def. 11) and state. We say that a trace reaches a configuration $\langle\rho, q\rangle$ for pattern $p$ iff $q_{0} \stackrel{\rho(\tau)}{\longrightarrow} q$ where $q_{0}$ and $\rightarrow$ are the initial state and transition relation of $p$.

Algorithm 1 gives an algorithm for computing the failing-edit-distance by computing the set of configurations reached by a trace. The algorithm uses a tolerance $\gamma$ to restrict the size of rewrites and therefore the algorithm will only find the edit-distance if it is below this tolerance. The algorithm uses a function failing that returns true if a final state is not reachable from the given state.

The use of $\gamma$ helps restrict the exponential blowup introduced by the nondeterminism of edit functions. Other optimisations that can reduce this blowup include restricting the number of edits allowed in a row and combining similar rewrites together.

\subsection{Example of computing failing-edit-distance}

Let us take the resource usage pattern introduced in Sec. 3.2 and consider the trace for the instantiation $[a \mapsto$ open, $b \mapsto$ use, $c \mapsto$ close $]$. Checking this pattern will fail on the second event as there is no $a$ transition from the second state. Two edit operations can be applied here - removal of the second event or addition of a close event immediately before the second open - this leads to two alternative configurations:

$$
\{\langle[(1,-)], 2\rangle,\langle[(1,+ \text { close })], 2\rangle\}
$$

We continue checking and fail again on the fifth event, the final use. Here there are also three edit operations that can be applied - removal of the event, addition of a open event or substitution of the use event with an open event. This leaves us with six final configurations:

$$
\left\{\begin{array}{c}
\langle[(1,-),(5,-)], 1\rangle,\langle[(1,-),(5,+ \text { open })], 2\rangle, \\
\langle[(1,-),(5, \% \text { open })], 2\rangle,\langle[(1,+ \text { close }),(5,-)], 1\rangle, \\
\langle[(1,+ \text { close }),(5,+ \text { open })], 2\rangle,\langle[(1,+ \text { close }),(5, \% \text { open })], 2\rangle
\end{array}\right\}
$$

Therefore, the instantiated pattern matches with failing-edit-distance 2.

\section{USING THE TRUE EDIT DISTANCE}

We now consider an approach that uses the true edit distance between the trace and language. We consider a technique that uses weighted transducers to compute the edit-distance between a trace and a finite automaton [2]. The general idea is that we model the trace and pattern as weighted transducers $T$ and $P$ and model the edit operations as a transducer $X$. The composition $T \circ X \circ P$ will capture the different ways that the trace can be rewritten to match the pattern and the minimal edit-distance is the shortest path to an accepting state.

\subsection{Weighted transducers}

A weighted transducer has transitions labeled with an input symbol, output symbol and weight - for this application we take weights as being 0 or 1. We allow $\epsilon$ input and output transitions that can be taken without consuming or producing a symbol.

DEFINITION 12 (WEIGHTED TRANSDUCER). A weighted transducer is a 5-tuple $T=\langle Q, \Sigma, \Delta, \delta, F\rangle$ where $Q$ is a finite set of states, $\Sigma$ is a finite input alphabet of symbols, $\Delta$ is a finite output alphabet of symbols, $\delta \subset Q \times(\Sigma \cup\{\epsilon\}) \times(\Delta \cup\{\epsilon\}) \times\{0,1\} \times Q$ is a finite set of transitions and $F \subseteq Q$ is a set of final states.

We translate traces into weighted transducers by creating a transition to a new state per event, adding self-looping $\epsilon$ transitions and only making the last state final. For example, the trace a.a.b.c.b would become the following weighted transducer where transitions are written input/output : weight. Note that we use a weight of 0 as there is no cost associated with following the trace.

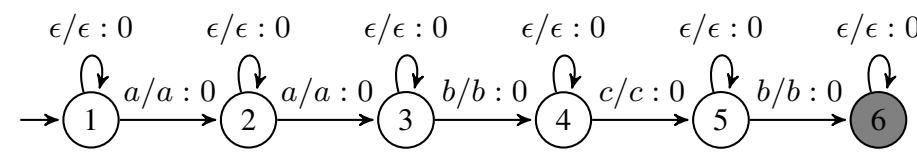

Patterns are translated by keeping the structure and labeling transitions with the same input and output symbols using a weight of 0 , and adding self-looping $\epsilon$ transitions

The edit transducer consists of a single state and looping transitions for each of the edit operations it can perform - for an alphabet of $\{a, b, c\}$ this would be as follows. Note how $\epsilon$ is used to model deletions and additions and all edit operations have a weight of 1 .

$$
a / a: 0, b / b: 0, c / c: 0,
$$

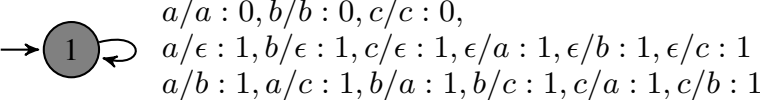




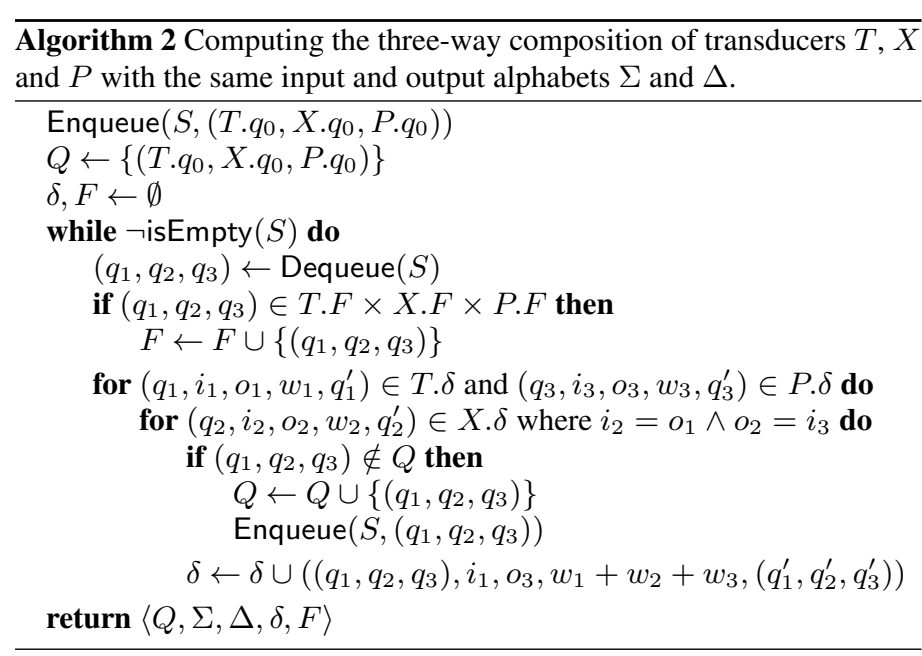

\subsection{Composition}

The composition $T \circ X$ of two transducers $T$ and $X$ considers all possible sequencing between strings of $T$ and strings $X$ i.e. if $a / b . a / c$ is a string of $T$ and $b / d . c / a$ is a string of $X$ then $a / d . a / a$ is a string of $T \circ X$. Here we consider a three-way composition i.e. $T \circ X \circ P$. We compute as a single operation for efficiency reasons - if we computed $T \circ X$ and then $(T \circ X) \circ P$ it is likely that $(T \circ X)$ would contain many superfluous transitions. An approach for doing this is presented in [1] and Algorithm. 2 gives an algorithm for three-way composition.

\subsection{An example of computing edit-distance}

Let us take the same example we used for the failing edit-distance i.e. the trace

$$
\text { open.open.use.close.use }
$$

and the resource usage pattern introduced in Sec. 3.2. For ease of presentation we translate the trace using $a$ for open, $b$ for use and $c$ for close. This gives us the trace used as an example in Sec. 5.1 above. We therefore already have our weighted transducer $T$. We then compute the weighted transducer $P$ for the resource usage pattern as follows.

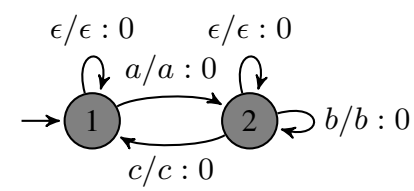

We now compute $T \circ X \circ P$, using the edit transducer $X$ presented in Sec. 5.1 above. This gives us the weighted transducer in Figure 1. We then use Djkistra's shortest path algorithm to find a shortest path between the initial state and an accepting trace. We indicate one such shortest path with a dashed line, this corresponds to the string $a / a . a / b . b / b . c / c . b / a$ with a weight of 2 . This gives two edits to our string - replacing the second open event with a use event and the last use event with an open event. Note that there are multiple paths with a weight of 2 here, and therefore multiple ways we can rewrite our trace.

A shortest path through the composition will always be at least as long as the trace and will give a rewrite by relating the projected trace back to the original trace. If a pattern checker is used then, instead of computing the shortest distance to an accepting state, for each pattern we compute the shortest distance to an accepting state labeled with that pattern.

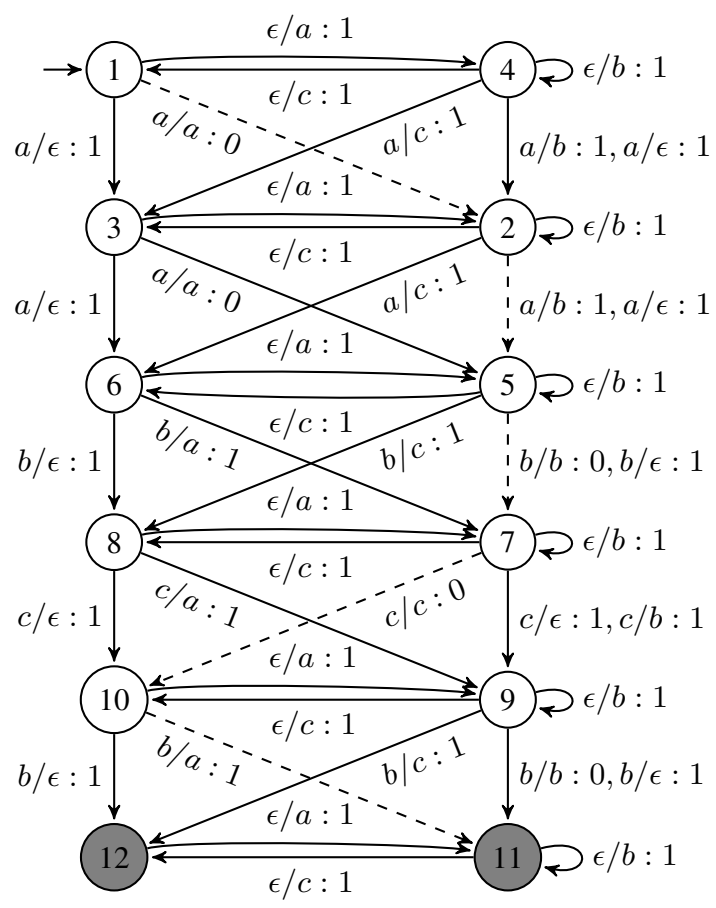

Figure 1: An example of the composition $T \circ X \circ P$

\section{COMBINING IMPERFECT PATTERNS}

The previous two sections presented two different techniques for extracting 'imperfect' patterns from imperfect traces. Each pattern is given a set of rewrites that tell us how to edit the input trace to make it match the pattern. When combining patterns we now need to consider these rewrites. In this section we present an approach for combining a set of imperfect patterns that are compatible i.e. have a set of rewrites that do not clash. We then discuss a saturation approach to producing a set of pattern combinations.

\subsection{The approach}

We first define what we mean by imperfect pattern. If we took an imperfect pattern as a pair of a pattern and its shortest rewrite then when combining two patterns we might find that these shortest rewrites are incompatible, but that if we had chosen, say, the second shortest rewrite we would be able to combine the two patterns. Therefore, we consider all rewrites up to a certain size for a pattern.

An imperfect pattern is a pair $\langle p, R\rangle$ where $p$ is a pattern and $R$ is a set of rewrites. In the case of the failing edit-distance approach $R$ is given by the reached configurations. In the case of true edit-distance approach $R$ is given by the language of the composition - therefore can be infinite, but in practice we use a breadth-first search to select the $k$-shortest paths.

A set of imperfect patterns $\left\{\ldots\left\langle p_{i}, R_{i}\right\rangle \ldots\right\}$ is compatible if there exists a set of rewrites $\left\{\ldots \rho_{i} \ldots \mid \rho_{i} \in R_{i}\right\}$ such that every pair of rewrites is compatible. Two rewrites are compatible if they do not attempt to make different rewrites at the same relevant points in a trace. We interpret no edit as an identity edit. A point in the trace is relevant to a rewrite if it is in the alphabet of the associated pattern. The edit-distance of $\left\langle p_{n}, R_{n}\right\rangle \cap$ $\ldots \cap\left\langle p_{n}, R_{n}\right\rangle$ is $\left|\rho_{1} \cup \ldots \cup \rho_{n}\right|$ i.e. the number of edits when all rewrites are combined. Therefore, given a set of compatible patterns we want to find the set of rewrites that minimizes this distance. 


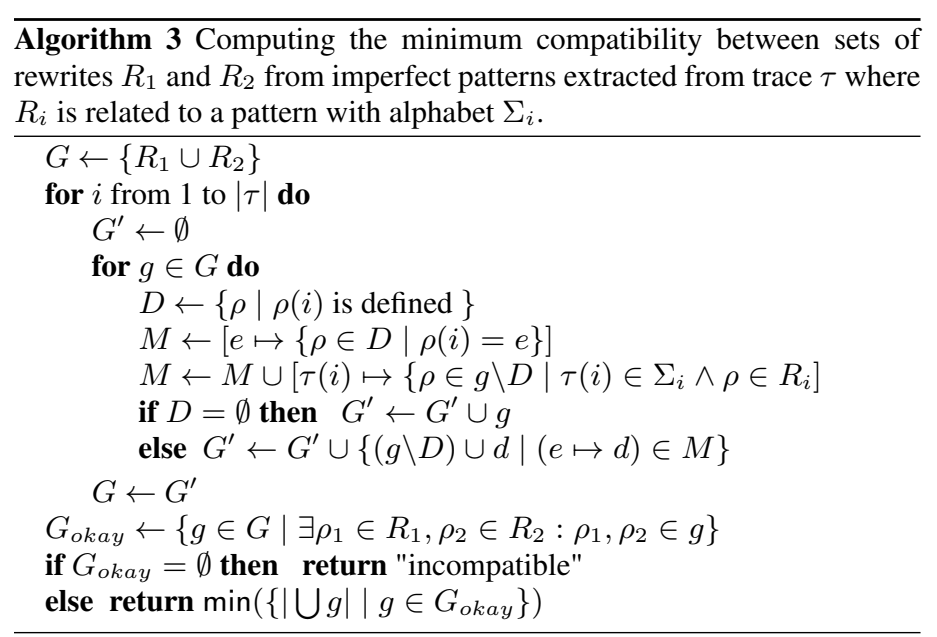

\subsection{Computing compatibility}

We compute the compatibility between two sets of rewrites $R_{1}$ and $R_{2}$ by taking the the set $R_{1} \cup R_{2}$ and repeatedly splitting it based on conflicts between rewrites and then checking that there is a set of rewrites with a rewrite in $R_{1}$ and $R_{2}$. An algorithm for computing compatibility is given in Algorithm 3. This can be extended to a set of sets of rewrites.

The algorithm will return "incompatible" if the two sets of rewrites are incompatible and the smallest number of edits that makes them compatible otherwise. Let min be the function that returns this minimum distance and is undefined otherwise.

\subsection{Saturating the set of patterns}

Given a set of imperfect patterns $P_{0}$ extracted from a trace we compute the $i$ th saturation of $P_{0}$ as follows, recalling that $\min \left(R_{1}, R_{2}\right)$ is only defined if $R_{1}$ and $R_{2}$ are compatible.

$$
P_{i+1}=\left\{\left\langle p_{1} \cap p_{2}, \min \left(R_{1}, R_{2}\right)\right\rangle \mid\left\langle p_{1}, R_{1}\right\rangle\left\langle p_{2}, R_{2}\right\rangle \in P_{i}\right\}
$$

In general, $\left|P_{i}\right|=\frac{1}{2}\left|P_{i-1}\right|\left(\left|P_{i-1}\right|-1\right)$. However, many combinations in $P_{i_{1}}$ will be trivial and can be removed. However, the saturation can grow exponentially. Let $P_{\infty}$ be the fixed-point of $P_{i}$ i.e. the set $P_{i}$ such that $P_{i+1}=P_{i}$. To make saturation practical we take the following steps:

- Limit - We place an upper limit on the saturation set i.e. $P_{3}$

- Prune - We filter out patterns if:

- Subsumption - they are subsumed by another pattern.

- Maximal alphabet - they do not use all symbols.

- Minimum distance - they are not within a small bound of the minimum edit distance used.

- Rank - We rank patterns by edit-distance and size.

\section{EXPERIMENTS}

In this section we explore our new technique by first applying it to a hypothetical code snippet and then carrying out an experiment to evaluate accuracy where we attempt to recreate a known specification from imperfect traces.

\subsection{Application to example code}

Consider the Java code in Figure 2. This gives a hypothetical method for sending an array of lines to an address by first connecting to that address, opening a stream, sending the lines and then closing the stream. This

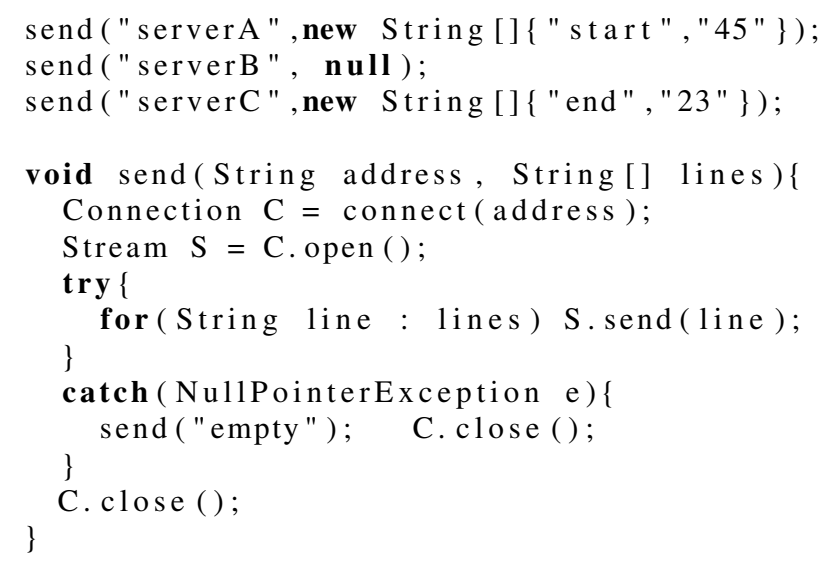

Figure 2: A hypothetical piece of Java code.

example contains a bug - in the case where a null array of lines is given the connection is closed twice.

Let us assume we execute the above code, which calls the method three times with different inputs, recording the occurrences of the connect, open, send and close events. The resulting trace would be as follows.

connect.open.send.send.close.connect.open.send.close.close. connect.open.send.send.close.

We now consider mining this trace with two patterns - the alternating pattern given in the introduction and the resource usage pattern given in Section 3.2. We take the alternating pattern first.

The following table gives the failing and true edit-distances (failing/true) for the above trace and the different instantiations of the alternating pattern - a '-' represents that no distance should be given (we do not consider the case where $a=b$ ) and an ' $\mathrm{x}$ ' represents that no distance is returned. The instantiation $[a \mapsto$ open, $b \mapsto$ connect $]$ does not have a failing edit-distance as it finishes in a non-final state that can be extended to a final state - this is one drawback of the failing edit-distance approach. For $[a \mapsto$ close, $b \mapsto$ connect $]$ and $[a \mapsto$ close, $b \mapsto$ open $]$ there is a shorter true edit-distance as this approach is allowed to make edits without failure - here removing the last event to bring the pattern into an accepting state. Note that all other distances are the same, this shows that in failing edit-distance can be a good approximation of true edit-distance.

\begin{tabular}{c|c|cccc} 
& & \multicolumn{4}{|c}{$\mathrm{a}$} \\
\hline & & connect & open & send & close \\
\hline \multirow{4}{*}{$\mathrm{b}$} & connect & - & $\mathrm{x} / 2$ & $3 / 3$ & $4 / 3$ \\
& open & $0 / 0$ & - & 3 & $4 / 3$ \\
& send & $2 / 2$ & $2 / 2$ & - & $4 / 3$ \\
& close & $1 / 1$ & $1 / 1$ & $3 / 3$ & -
\end{tabular}

For one case, $[a \mapsto$ connect, $b \mapsto$ open] there is a distance of 0 - this is because this instantiated pattern matches the trace exactly. If we consider the two cases where there is an edit-distance of 1 and look at the rewrite generated we see that all of these produce the same rewrite - the removal of the ninth event (the second close).

Combining the three instantiated patterns with an edit distance of 0 or 1 we get the following pattern.

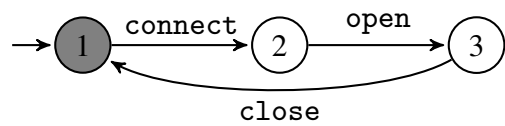


Now let us consider the resource usage pattern. The following table gives the failing and true edit distances as before - with each entry in the table representing the $c$ dimension using a 4-tuple. Here, again, computed distances are the same but the true edit-distance approach generates some distances where the failing edit-distance approach does not.

\begin{tabular}{cc|cccc} 
& & \multicolumn{4}{|c}{ a } \\
& & connect & open & send & close \\
\hline \multirow{4}{*}{$\mathrm{b}$} & connect & $(-,-,-,-)$ & $(-,-, 5 / 5,4 / 4)$ & $(-, 3 / 3,-, 5 / 5)$ & $(-, 2 / 2,4 / 4,-)$ \\
& open & $(-,-, 2 / 2,1 / 1)$ & $(-,-,-,-)$ & $(5,-,-, 5)$ & $(5 / 5,-, 4 / 4,-)$ \\
& send & $(-, 4 / 4,-, 1 / 1)$ & $(1 / 1,-,-, 1 / 1)$ & $(-,-,-,-)$ & $(\mathrm{x} / 6, \mathrm{x} / 6,-,-)$ \\
& close & $(-, 4 / 4,5 / 5,-)$ & $(1 / 1,-, 5 / 5,-)$ & $(3 / 3,3 / 3,-,-)$ & $(-,-,-,-)$
\end{tabular}

There are five instantiations with an edit-distance of 1 , but they represent different rewritings of the trace. One set removes the ninth event (as before) and one set removes the first connect event, therefore they are incompatible. When combined they give the following respectively:
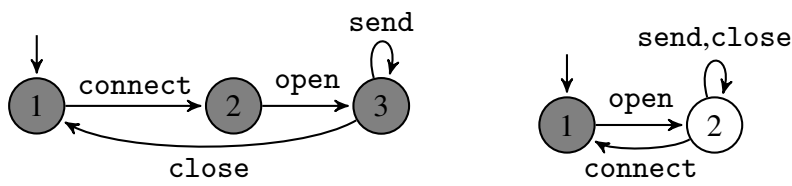

The rewrite for first pattern is compatible with the rewrite for pattern extracted using the alternation pattern and we can combine these patterns to form a final specification, which is the same as the one on the left above, but with only the initial state accepting.

\subsection{An accuracy experiment}

Before we begin we should note that this experiment is not fully measuring the expected usage of this technique as there is no manual inspection of the produced specifications. We evaluate the accuracy of our approach by generating traces from the following specification for the Lucene tool described in [5].

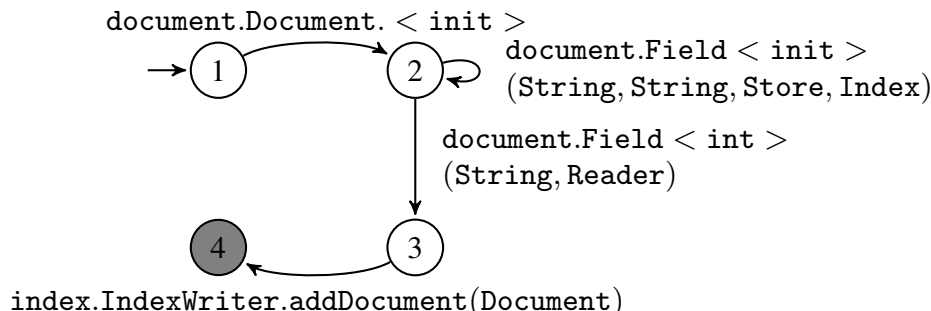

index.IndexWriter.addDocument(Document)

We generate imperfect traces by first generating perfect traces and then randomly editing events according to some noise level (probability). We then pass these traces to our techniques and test the resulting patterns for accuracy using a set of perfect traces generated from the specification. Table 1 gives the average results over three runs. For each approach it reports the average accuracy, the minimum edit required to produce a pattern with maximum accuracy, the time taken for checking and then saturation and the size of the pruned 3-saturated set. Experiments were carried out with a range of trace lengths and noise levels and different $\gamma$ for failing and $k$-shortest paths for perfect.

Every experiment with a non-empty $P_{3}$ produced at least one pattern with perfect accuracy. As expected, with zero noise we achieve perfect accuracy. The reason we saw empty $P_{3}$ sets for Failing was that the $\gamma$ was not high enough and for Perfect it was due to the relevant rewrites not appearing in the top $k$-shortest sets. These parameters can be increased, but they currently have a high impact on running times. As expected, as noise increases accuracy generally decreases and in general the larger $\gamma$ or $k$ the better accuracy.

Generally checking times are very fast, with saturation dominating the process. The main cost in saturation is the computation of comparability between rewrites - which is why we saw the highest saturation times with Failing-5. Further work should consider methods for optimising this process and trimming the set of rewrites considered.

It is clear that saturation of this kind is too costly. If we combine this approach with the open automata of [17] we would combine together compatible patterns directly, without saturation. Alternatively, the introduction of specific combination rules would reduce the cost of saturation.

\section{RELATED WORK}

We consider alternative techniques that mine specifications from runtime traces. A recent survey paper [18] gives a good overview of the field. Here we focus on how techniques deal with imperfect traces, in particular we are interested in automata-based pattern mining approaches.

Ammons et al. [3] developed an early approach that used a probabilistic finite automata learner from the field of grammar inference and requires the alphabet of the inferred specification to be known beforehand. Imperfect traces require human experts to check violations of the inferred specification in a coring phase. Lo et al. [14] extend this approach - one extension that is relevant here is the introduction of a stage that attempts to filter out erroneous traces before learning. In contrast we attempt to use this information to extract a specification and identify the error.

Techniques that use frequent-itemset mining (i.e. [12]) and closed frequent sequential pattern mining (i.e. [15]) rely on computing support and confidence values where support reflects the level of imperfection, and therefore can handle imperfect traces. However, the properties extracted are not as strict as automata-based specifications as in the first case symbols are only related by frequent association, not order, and in the second the ordering relation is simple. These techniques scale very well and require minimal information to be provided.

The automata-based pattern-mining technique was first used by Engler et al. [4]. They focus on the alternating pattern $(a b)^{*}$ and deal with imperfect traces by counting the number of times that $a$ and $b$ occur together in order, and $a$ occurs without $b$ and compute the likelihood that they form a specification. Goues and Weimer [8] extend this approach with techniques for pruning false positives by examining the source code.

Yang et al. [19] introduced a template-based technique focusing on extracting specifications from imperfect traces. They use the alternating pattern and deal with imperfect traces by partitioning a trace into sequences of one event followed by another, i.e. $a^{+} b^{+}$, performing mining on each subtrace and then counting the number of subtraces the pattern holds for. This is similar to restarting the pattern on failure but allows for a larger range of failures. They also introduce a chaining heuristic for combining their alternating patterns.

Gabel and Su. [5, 6] extend this approach by introducing a symbolic method for specification mining using binary decision diagrams and the Javert tool that uses two patterns $(a b)^{*}$ and $\left(a b^{*}\right)^{*}$ and composition rules based on automata combination to extract large patterns. They deal with imperfect traces by restarting a pattern at the initial state on failure. In [7] they extend this approach to infer and enforce temporal properties at runtime over a finite window, thus detecting potential bugs at runtime.

$\mathrm{Li}$ et al. [11] extend this approach to mine specifications with timing bounds and more complex pattern composition rules, but cannot handle imperfect traces. Instead their focus is on mining specifications from 


\begin{tabular}{|c|c|c|c|c|c|c|c|c|c|c|c|c|c|c|c|c|c|}
\hline \multirow{2}{*}{$\begin{array}{l}\text { Trace } \\
\text { length }\end{array}$} & \multirow{2}{*}{$\begin{array}{l}\text { Noise } \\
\text { level }\end{array}$} & \multicolumn{4}{|c|}{ Failing-2 } & \multicolumn{4}{|c|}{ Failing-5 } & \multicolumn{4}{|c|}{ Perfect-2 } & \multicolumn{4}{|c|}{ Perfect-20 } \\
\hline & & A & $\mathrm{E}$ & Time & $P_{3}$ & A & $\mathrm{E}$ & Time & $P_{3}$ & A & $\mathrm{E}$ & Time & $P_{3}$ & A & $\mathrm{E}$ & Time & $P_{3}$ \\
\hline 10 & 0.0 & 1.0 & 0 & $0.06,0.84$ & 36 & 1.0 & 0 & $0.06,34.7$ & 34 & 1.0 & 0 & $0.11,0.13$ & 36 & 1.0 & 0 & $0.05,0.11$ & 34 \\
\hline 10 & 0.05 & 0.48 & 1 & $0.01,0.96$ & 11 & 0.83 & 3 & $0.01,195$ & 1 & 0.86 & 1 & $0.04,0.05$ & 4 & 0.78 & 1 & $0.03,0.03$ & 4 \\
\hline 10 & 0.1 & 0.58 & 2 & $0.01,2.22$ & 2 & 0.80 & 3 & $0.01,172$ & 14 & 0.68 & 2 & $0.03,0.06$ & 13 & 0.87 & 2 & $3,0.04$ & 4 \\
\hline 100 & 0.0 & 1.0 & 0 & $0.09,1.64$ & 35 & 1.0 & 0 & $0.09,1.47$ & 36 & 1.0 & 0 & $0.46,0.27$ & 33 & 1.0 & 0 & $0.26,0.16$ & 34 \\
\hline 100 & 0.05 & 0.33 & 1 & $0.01,4.96$ & 1 & 0.53 & 4 & $0.01,476$ & 2 & 0.66 & 1 & $0.27,0.17$ & 1 & 0.0 & - & $0.29,0.12$ & 0 \\
\hline 1000 & 0.0 & 1.0 & 0 & $0.17,11.5$ & 35 & 1.0 & 0 & $0.17,12.4$ & 32 & 1.0 & 0 & $3.29,5.13$ & 33 & 1.0 & 0 & $3.09,0.70$ & 35 \\
\hline 1000 & 0.01 & 0.0 & - & $0.16,2.48$ & 0 & 0.33 & 2 & $0.16,1382$ & 1 & 0.0 & - & $3.48,3.67$ & 0 & 0.16 & 1 & $3.11,3.5$ & 2 \\
\hline
\end{tabular}

Table 1: Results from accuracy experiment. $A=$ accuracy. $E$ = edits. Time gives checking and saturation time separately in seconds.

perfect traces and using these to detect bugs in imperfect ones.

Finally, recent techniques $[9,16,17]$ consider the parametric case where traces contain data i.e. open(12).open(45).close(12). close (45). Whilst some approaches use ad-hoc methods to deal with context, these focus on slicing the trace based on this data and extracting traces from the resulting data-free traces. The work in [9] extends the approach taken by [3] and therefore use the same coring technique to deal with imperfect traces and [16] uses the notions of support and confidence from data mining.

\section{CONCLUSION}

This paper has introduced a new approach for mining specifications from imperfect traces. Two techniques are introduced that use the notion of edit-distance to compute the number of changes that would have to be made to a trace for a pattern to hold and a notion of when it safe to combine two imperfect patterns is given. We also demonstrate the process by applying it to a small code snippet and then measure the accuracy of the approach using traces generated from a known specification.

This technique not only produces specifications, but also a description of how a program should be updated to make the specification hold. This would be useful in bug detection and location but a case study is required to establish applicability.

Further work is required to improve the efficiency and applicability of the approach. This should involve incorporating existing techniques, for example the symbolic mining technique of [6], and the composition rules of $[5,11]$. We also plan on combining this approach with the author's pattern-mining approach taken in [17], which targets a specific alphabet of events to extract a parametric specification. This approach uses socalled open automata that means that all extracted patterns can be sound combined to form a specification. Therefore, we would be able to use pattern combination directly, rather than introducing pattern combination rules. A further area of interest is the use of edit-distance as a fitness function in evolutionary techniques used to "evolve" a specification.

\section{REFERENCES}

[1] C. Allauzen and M. Mohri. 3-way composition of weighted finite-state transducers. In Proceedings of the 13th international conference on Implementation and Applications of Automata, CIAA '08, pages 262-273, Berlin, Heidelberg, 2008. Springer-Verlag.

[2] C. Allauzen and M. Mohri. Linear-space computation of the edit-distance between a string and a finite automaton. CoRR, abs/0904.4686, 2009.

[3] G. Ammons, R. Bodík, and J. R. Larus. Mining specifications. SIGPLAN Not., 37(1):4-16, Jan. 2002.

[4] D. Engler, D. Y. Chen, S. Hallem, A. Chou, and B. Chelf. Bugs as deviant behavior: a general approach to inferring errors in systems code. SIGOPS Oper. Syst. Rev., 35(5):57-72, Oct. 2001.

[5] M. Gabel and Z. Su. Javert: fully automatic mining of general temporal properties from dynamic traces. In Proceedings of the 16th ACM SIGSOFT International Symposium on Foundations of software engineering, SIGSOFT '08/FSE-16, pages 339-349, New York, NY, USA, 2008. ACM.

[6] M. Gabel and Z. Su. Symbolic mining of temporal specifications. In ICSE '08: Proceedings of the 30th international conference on Software engineering, pages 51-60, New York, USA, 2008. ACM.

[7] M. Gabel and Z. Su. Online inference and enforcement of temporal properties. In Proceedings of the 32nd ACM/IEEE International Conference on Software Engineering - Volume 1, ICSE '10, pages 15-24, New York, NY, USA, 2010. ACM.

[8] C. Goues and W. Weimer. Specification mining with few false positives. In Proceedings of the 15th International Conference on Tools and Algorithms for the Construction and Analysis of Systems., TACAS '09, pages 292-306, Berlin, Heidelberg, 2009. Springer-Verlag.

[9] C. Lee, F. Chen, and G. Roşu. Mining parametric specifications. In Proceeding of the 33rd International Conference on Software Engineering (ICSE'11), pages 591-600. ACM, 2011.

[10] V. Levenshtein. Binary Codes Capable of Correcting Deletions, Insertions and Reversals. Soviet Physics Doklady, 10:707, 1966.

[11] W. Li, A. Forin, and S. A. Seshia. Scalable specification mining for verification and diagnosis. In DAC '10: Proceedings of the 47th Design Automation Conference, pages 755-760, New York, NY, USA, 2010. ACM.

[12] Z. Li and Y. Zhou. Pr-miner: automatically extracting implicit programming rules and detecting violations in large software code. SIGSOFT Softw. Eng. Notes, 30(5):306-315, Sept. 2005.

[13] D. Lo, K. Cheng, and J. Han. Mining Software Specifications: Methodologies and Applications. Chapman and Hall/CRC Data Mining and Knowledge Discovery. Taylor \& Francis Group, 2011.

[14] D. Lo and S.-C. Khoo. Smartic: towards building an accurate, robust and scalable specification miner. In Proceedings of the 14th ACM SIGSOFT international symposium on Foundations of software engineering, SIGSOFT '06/FSE-14, pages 265-275, New York, USA, 2006. ACM.

[15] D. Lo, S.-C. Khoo, and C. Liu. Mining temporal rules for software maintenance. J. Softw. Maint. Evol., 20(4):227-247, July 2008.

[16] D. Lo, G. Ramalingam, V. P. Ranganath, and K. Vaswani. Mining quantified temporal rules: Formalism, algorithms, and evaluation. Sci. Comput. Program., 77(6):743-759, 2012.

[17] G. Reger, H. Barringer, and D. Rydeheard. A pattern-based approach to parametric specification mining. In Proceedings of the 28th IEEE/ACM International Conference on Automated Software Engineering, November 2013. To appear.

[18] M. P. Robillard, E. Bodden, D. Kawrykow, M. Mezini, and T. Ratchford. Automated api property inference techniques. IEEE Transactions on Software Engineering, 39(5):613-637, 2013.

[19] J. Yang, D. Evans, D. Bhardwaj, T. Bhat, and M. Das. Perracotta: mining temporal api rules from imperfect traces. In ICSE '06: Proceedings of the 28th international conference on Software engineering, pages 282-291, New York, NY, USA, 2006. ACM. 